\title{
Aplicação da escala de Herth e suas correlações em transplante de células-tronco
}

\section{hematopoiéticas}

\author{
Aplicattion of the scale Herth and correlations in hematopoietic stem cell-transplantation \\ Aplicación Herth y correlaciones em el transplante de células-madre hematopoyéticas
}

Wabison Júnior Fernandes dos Santos

ORCID: https://orcid.org/0000-0002-6322-9529

Hospital Israelita Albert Einstein de São Paulo, Brasil

E-mail: wabison.santos@einstein.br

Maria Aparecida Machado

ORCID: https://orcid.org/0000-0002-6176-0746

Hospital Israelita Albert Einstein de São Paulo, Brasil

E-mail: maria.amachado@einstein.br

Gercilene Cristina Silveira

ORCID: https://orcid.org/0000-0002-1642-6917

Faculdades Integradas de Jaú, Brasil

Hospital Amaral Carvalho, Brasil

E-mail: ger_silveira@hotmail.com

Andressa Fabri de Souza Santos

ORCID: https://orcid.org/0000-0002-4579-281X

Hospital Regional de Presidente Prudente, Brasil

E-mail: andressafabri98@gmail.com

Talita Oliveira de Lima

ORCID: https://orcid.org/0000-0002-6617-6514 Hospital das Clínicas da Faculdade de Medicina de Botucatu, Brasil E-mail: talima.2111@gmail.com

Ariane Pelossi de Oliveira

ORCID: https://orcid.org/0000-0002-2248-6174

Unimed Presidente Prudente, Brasil

E-mail: arianepelossioliveira@gmail.com

Murilo Meidas Ferrer

ORCID: https://orcid.org/0000-0002-7195-4640

Universidade do Oeste Paulista de Presidente Prudente, Brasil

E-mail: muriloferrer@yahool.com.br

Bruna Larissa Cápera da Silva

ORCID: https://orcid.org/0000-0002-7399-0899

Hospital Amaral Carvalho de Jaú, Brasil

E-mail: brunna.capera@ @otmail.com

Eliana Marangoni

ORCID: https://orcid.org/0000-0003-0265-4254

Universidade do Oeste Paulista Unoeste de Jaú, Brasil E-mail: eliguidugli@hotmail.com

Elaine Cristina Negri Santos

ORCID: https://orcid.org/0000-0001-8665-1936

Universidade do Oeste Paulista de Presidente Prudente, Brasil

E-mail: elainenegrisantos@gmail.com

Karina Alexandra Batista da Silva Freitas

ORCID: https://orcid.org/0000-0002-3413-8468

Hospital das Clínicas da Faculdade de Medicina de Botucatu, Brasil

E-mail:k.freitas@unesp.br

\begin{abstract}
Resumo
A esperança é definida como uma perspectiva positiva em relação ao futuro, inspirando coragem para enfrentar o medo. Apesar de não ser considerada uma forma de cura, ela motiva o paciente a enfrentar a doença em um aspecto positivo. Este estudo objetiva avaliar o nível de esperança de vida dos pacientes adultos no período pré - transplante de células-tronco hematopoiéticas e sua relação com resultados no período de 100 dias pós- procedimento. Trata-se de um estudo de natureza observacional descritiva, com abordagem quantitativa e recorte transversal, realizado em um ambulatório especializado no serviço de transplante de medula óssea, no período do mês de abril a dezembro de 2020. Participaram deste estudo um amostral de 82 pacientes, sendo 38 (46\%) coletados Pré-Transplante de Células TroncoHematopoiéticas (TCTH) e 44 (54\%) Pós- Transplante de Células Tronco-Hematopoiéticas (TCTH), utilizando-se a escala de esperança de Herth (EEH). Quanto aos resultados a amostra apresentou nível de esperança considerado alto
\end{abstract}


$(41,9)$ pontos no grupo pré, seguido do grupo pós $(40,7)$ pontos. Conclui-se que os pacientes em tratamento pré e pós transplante apresentaram altos níveis de esperança durante todo o processo de tratamento. Entretanto, foi mostrado que os indivíduos que realizam prática religiosa como estratégia para o enfrentamento da doença, apresentaram altos níveis de esperança. Os dados deste estudo apontam que se torna relevante aos profissionais de enfermagem encorajar cuidados que estimulem os mecanismos positivos das crenças, elevando os níveis de esperança.

Palavras-chave: Esperança de vida; Crenças; Transplante de medula óssea; Avaliação em enfermagem.

\begin{abstract}
Hope is defined as a positive outlook on the future, inspiring courage to face fear. Although not considered a form of cure, it motivates the patient to face the disease in a positive way. This study aims to assess the level of life expectancy of adult patients in the pre - hematopoietic stem cell transplantation period and its relationship with results within 100 days post-procedure. This is a descriptive observational study, with a quantitative approach and crosssectional, carried out in an outpatient clinic specializing in the bone marrow transplant service, from April to December 2020. A sample of 82 patients participated in this study, being 38 (46\%) collected Pre-Hematopoietic Stem Cell Transplantation (HSCT) and 44 (54\%) Post-Hematopoietic Stem Cell Transplantation (HSCT), using the Herth Hope Scale (EEH). As for the results, the sample showed a level of hope considered high (41.9) points in the pre group, followed by the post group (40.7) points. It is concluded that patients undergoing pre- and post-transplant treatment had high levels of hope throughout the treatment process. However, it was shown that individuals who perform religious practice as a strategy to deal with the disease, showed high levels of hope. The data from this study indicate that it is relevant for nursing professionals to encourage care that estimulates the positive mechanisms of beliefs, increasing levels of hope.
\end{abstract}

Keywords: Life expectancy; Beliefs; Bone marrow transplant; Nursing assessment.

\title{
Resumen
}

La esperanza se define como una visión positiva en relación con el futuro, que inspira coraje para enfrentar el miedo. Aunque no se considera una forma de cura, motiva al paciente a afrontar la enfermedad de forma positiva. Este estudio tiene como objetivo evaluar el nivel de esperanza de vida de los pacientes adultos en el período previo al trasplante de células madre hematopoyéticas y su relación con los resultados dentro de los 100 días posteriores al procedimiento. Se trata de un estudio observacional descriptivo, de abordaje cuantitativo y transversal, realizado en un ambulatorio especializado en el servicio de trasplante de médula ósea, de abril a diciembre de 2020. En este estudio participó una muestra de 82 pacientes., Siendo 38 (46\%) recogieron trasplante de células madre prehematopoyéticas (TCMH) y $44(54 \%)$ trasplante de células madre poshematopoyéticas (TCMH), utilizando la escala de esperanza de Herth (EEH). En cuanto a los resultados, la muestra mostró un nivel de esperanza considerado alto $(41,9)$ puntos en el grupo pre, seguido del grupo post $(40,7)$ puntos. Se concluye que los pacientes sometidos a tratamiento antes y después del trasplante mostraron altos niveles de esperanza durante todo el proceso de tratamiento. Sin embargo, se demostró que las personas que realizan la práctica religiosa como estrategia para hacer frente a la enfermedad, mostraron altos niveles de esperanza. Los datos de este estudio indican que es relevante que los profesionales de enfermería fomenten cuidados que fomenten los mecanismos positivos de las creencias, elevando los niveles de esperanza.

Palabras clave: Esperanza de vida; Creencias; Transplante de medula ósea; Evaluación en enfermería.

\section{Introdução}

A esperança é definida como uma perspectiva positiva de vida em relação ao futuro que inspira coragem para enfrentar o medo, sendo vivenciada de uma forma pessoal e única. Apesar de não ser considerada uma forma de cura, ela motiva o enfermo a agir e enfrentar a doença de forma diferente em um aspecto positivo. Quando relacionada a fenômenos sobrenaturais, como crenças e fé, pode ser capaz de proporcionar mudanças de vida para o paciente com a doença diagnosticada (Sartore \& Grossi, 2008).

O termo esperança, de acordo com a sua etimologia, apresenta um significado amplo relacionado à espiritualidade e crenças. A palavra esperança vem do latim: sperare. Sentimento que leva o homem a olhar para o futuro, considerando-o portador de condições melhores que as oferecidas pelo presente, de tal sorte que a luta pela vida e os sofrimentos são enfrentados como contingências passageiras na marcha para um fim mais alto e de maior valor. (...) No pensamento grego, Platão, designa "a grande e bela esperança" num além depois da morte. No pensamento romano, a palavra "spes" designava somente o momento feliz. A Esperança faz com que o nosso pensamento ultrapasse tempo e espaço e penetre na imensidão do espaço infinito. Assim, de posse desta virtude, esquecemo-nos momentaneamente das dores, dos sacrifícios, das doenças, das 
dificuldades, e lembramo-nos somente da felicidade regida pela paz e tranquilidade de nossas tensões. Isso não é utopia, é a dimensão do eu que se transcende a si mesmo rumo à espiritualidade superior (Tempesta, 2015, não paginado).

A esperança, advinda da espiritualidade, que pode ser expressa por meio da religião, apresenta significativa influência na forma como a pessoa se relaciona com a sua enfermidade, isto é, quando fornecido um ambiente adequado para sua expressão, favorece a compreensão sobre os riscos e benefícios de procedimentos, bem como adesão a tratamentos medicamentosos e não medicamentosos. À vista disso, há pacientes que possuem comportamento religioso e demonstram maiores níveis de esperança frente ao seu quadro, comparado àqueles que não expressam (Carvalho et al., 2021; Okuma et al., 2021).

No que diz respeito à esperança, enquanto construto, não possui uma definição universal, mas existe uma concordância de que é um evento multidimensional que perpassa por fatores religiosos, espirituais, orientação positiva para o futuro, ausência de fatores aversivos agudos e/ou frequentes, entre outros (Menezes et al., 2021). Desse modo, esta virtude tem sido amplamente averiguada em pacientes oncológicos e/ou que recebem cuidados paliativos (Grandizoli et al., 2017; Li et al., 2018; Sette \& Capitão, 2018).

O estudo transversal quantitativo de Wakiuchi et al., (2015), que tinha como finalidade verificar o nível de esperança de 60 pacientes no início e no final do tratamento quimioterápico, mediante a Escala de Esperança de Herth, não encontrou diferenças significativas entre os níveis de esperança no início e com longo tempo de tratamento quimioterápico. Entretanto, atribuíram que os aspectos que influenciaram a elevar os graus de expectativas foram: maior nível de escolaridade, ausência de metástases e a inexistência de dores severas ou moderadas.

Já a investigação transversal de Schuster et al., (2015), que avaliou esperança e depressão em 96 pacientes submetidos à quimioterapia no sul do Brasil, identificou que o grau de esperança dos enfermos depressivos era menor comparado aos não depressivos, assim como os que referiram prática religiosa e maior nível de escolaridade e, corroborando Wakiuchi et al., (2015), obtiveram maior pontuação na EEH ao apresentar maior nível de esperança.

Quando um indivíduo recebe o diagnóstico da doença é provável observar o impacto psicológico e emocional neste. O doente pode perder a expectação, as forças de enfrentamento, os estímulos e os objetivos de vida, além de fomentar em sua mente o pensamento de morte, podendo até mesmo atingir um quadro depressivo e contribuir para o agravamento da patologia. A observação dos profissionais de saúde no estado emocional do paciente é fundamental, uma vez que esses especialistas, ao oferecerem suporte emocional e detalhes sobre o tratamento, têm a capacidade de identificar evidências do nível de esperança (Eloia et al., 2021).

O diagnóstico de câncer oferece a capacidade de influenciar e provocar mudanças na vida do enfermo em relação ao aspecto biológico, fisiológico e social. Por meio dessa visão o paciente transfigura sua perspectiva de vida, pois a doença altera seu cotidiano e então será necessário se adaptar às novas mudanças (Franco et al., 2021).

As mutações em relação ao aspecto biológico se dão pelo surgimento de sintomas colaterais, como perda de peso, presença de nódulos e perda de cabelo, provocados por tratamentos longos com radioterapia, quimioterapia ou mutilações (Lima et al., 2020).

Em variadas situações de adoecimento, em algum momento, o Transplante de Células-Tronco Hematopoiéticas (TCTH) torna-se a opção mais viável de tratamento, como em doenças hematológicas malignas, hereditárias, imunológicas, metabólicas e oncológicas. Esse procedimento encontra-se em franca expansão e alcança cada vez mais resultados positivos, devido, principalmente, aos avanços tecnológicos que representam sua potencialidade (Gomes et al., 2019).

O TCTH é um procedimento longo e composto por fases distintos sendo durante a internação os cuidados diretos são realizados pela equipe de saúde em ambiente hospitalar, já após a alta o domicílio passa a representar o ambiente fundamental de zelo. Sendo assim, o cuidador familiar que responde pelas decisões permanece, a maior parte do tempo, com o paciente e se 
encarrega da grande parte das ações de cautela, mesmo durante o período de tratamento ambulatorial que se segue à alta hospitalar (Belo et al., 2020).

Entende-se que no contexto social aquele que enfrenta uma enfermidade passa por inúmeras modificações em sua rotina, como por exemplo: maior dependência dos cuidadores, alteração do estilo de vida, alterações corporais e isolamento das pessoas. Todos esses fatores podem transmudar sua prospectiva de vida ao desenvolver um sofrimento emocional e demonstrar sintomas de depressão, rebaixando o nível de esperança e amplificando a insatisfação com a estética. Isso pode interferir no processo de tratamento ou ainda piorar o quadro clínico (Silva et al., 2021).

Vale ressaltar que no processo do cuidado o acompanhamento dos familiares e o vínculo com a equipe de enfermagem é imprescindível, pois auxilia na comunicação do diagnóstico que, realizado de modo claro, oferece uma liberdade ao paciente e à família para dialogar sobre a doença, além de esclarecer a forma como será o tratamento. É nesse momento que o enfermo e seus parentes esclarecem suas dúvidas, expõem seus sentimentos, medos, inseguranças, questionamentos quanto ao tratamento e custos, entre outros. O doente necessitará de todo apoio possível para não amplificar o sentimento de desesperança (Freitas et al., 2021).

Durante a entrevista com aquele que está enfrentando a doença é importante identificar se há fatores que estejam diminuindo o grau de expectativa, sendo eles: depressão, ansiedade, estresse, desânimo, desinteresse, irritação, abatimento e diminuição do otimismo. Esses são os motivos que alteram toda a perspectiva de vida do paciente e pode agravar sua doença, dificultando o atendimento e a recuperação (Menezes et al., 2021).

A manutenção da esperança do paciente frente ao tratamento pode auxiliar no enfrentamento da doença. Deste modo mostra-se a importância de avaliar constantemente a esperança do paciente que irão ser submetidos ao TCTH para subsidiar o planejamento da intervenção de enfermagem bem como a capacitação dos profissionais de saúde para lidar com situações de crise e manutenção da qualidade de vida. O objetivo deste estudo é avaliar o nível de esperança de vida dos pacientes, adultos, no período pré - TCTH e sua relação com resultados observados no período de 100 dias pós- procedimento.

\section{Metodologia}

Estudo quantitativo, transversal, observacional descritivo.

Este estudo foi submetido e aprovado pelo comitê de Ética em Pesquisa do Hospital Amaral Carvalho (HAC), Protocolo: 025199/2020, CAAE: 30118020.8.0000.5434. Conforme previsto na Resolução CNS 466/2012, os sujeitos foram convidados a participar do estudo, seu aceite foi formalizado mediante a assinatura do Termo de Consentimento Livre e Esclarecido (TCLE) (APÊNDICE A). Foi mantido o anonimato dos mesmos e a confidencialidade dos dados coletados, foram mantidos em todas as fases da pesquisa, garantindo que não haverá prejuízos aos participantes.

O presente estudo foi desenvolvido na unidade de Transplante de Medula Óssea do Hospital Amaral Carvalho (HAC) situado na cidade de Jaú.

Foram convidados para colaborar com este estudo um total de aproximadamente 82 pacientes com diagnóstico de câncer e ou outras doenças graves com indicação de transplante, cadastrados e acompanhados no serviço. Os critérios de inclusão para cooperar com o estudo foram: ter diagnóstico comprovado, com recomendação de TCTH: Alogênico aparentado, não aparentado, haploidêntico e autólogo, estar em tratamento ou acompanhamento no serviço, ter idade igual ou superior a 18 anos e que apresentaram condições físicas e cognitivas para a contribuição nas entrevistas.

Para a coleta de dados foi estabelecido contato com a (o) enfermeiro (a) responsável pelo trabalho determinando o dia e horário de atendimento dos pacientes em tratamento e acompanhamento. A coleta de dados foi realizada por meio da entrevista, na unidade ambulatório do serviço de transplante de medula óssea, no período do mês de Abril a Dezembro de 2020. 
Assim sendo, foi realizada a aplicação da escala de esperança de Herth que foi traduzida e validada para língua portuguesa por Sartori \& Grossi, (2008), (ANEXO A), onde esta por sua vez, é composta por 12 itens escritos de forma afirmativa e a graduação dos mesmos ocorre por escala tipo Likert de 4 pontos, variando de concordo completamente, indicado por 4 , a discordo completamente, apontado por 1 . Os itens três e seis possuem o escore invertido. O escore total varia de 12 a 48, sendo que quanto maior o escore, mais alto o nível de esperança.

Os dados foram digitados duplamente em planilha de Excel e avaliados utilizando o Software: SPSS versão 23. Sendo aplicados, por meio do teste de Kolmogorov-Smirnov, os seguintes testes estatísticos: a verificação e a distribuição da amostra em paramétrico e não paramétrico. A finalidade é comparar os valores do questionário Herth entre duas variáveis ao empregar o teste T não pareado. Para a comparação dos valores do questionário Herth entre mais de duas variáveis será utilizado o OneWay ANOVA com pós-teste de BonFerroni. Será empregada a correlação de Pearson para verificar a correspondência entre o número de filhos e a pontuação do questionário de Herth. Para os testes estatísticos utilizaremos nível de significância de 95\%, considerados estatisticamente significativos os valores de $\mathrm{p}<0,05$. E desse modo foi feito a correlação com os resultados.

\section{Resultados e Discussão}

A amostra foi composta por 82 pacientes, sendo 38 (46\%) coletados Pré-TCTH e 44 (54\%) Pós-TCTH sendo que a maioria dos participantes entrevistados $53 \%$ é do sexo masculino, sendo $53 \%$ branco e deste $46 \%$ são casados. Ao se levantar os dados sobre as práticas religiosas $61 \%$ são católico sendo que a maioria sendo um total de $65 \%$ seguem uma prática religiosa.

Em relação ao nível de escolaridade dos participantes, quando se buscou saber se o entrevistado estava estudando no momento atual ou se se já tinha uma formação, o que se obteve foram as seguintes informações: 13 entrevistados (13\%) disseram ter nível superior completo, 1 (1\%) nível superior incompleto, 32 (39\%) ensino médio completo, 09 (11\%) ensino médio completo incompleto, 1 (1\%) nível superior incompleto, 10 (12\%) ensino fundamental completo, 17 (21\%) ensino fundamental incompleto, quanto aos dados de escolaridade a prevalência e de participantes com ensino médio completo.

Quando abordado a renda familiar dos entrevistados observa-se que média do participante é de até dois salários mínimos sendo que no quesito moradia dos 82 entrevistados 59 deste residem em casa própria.

Os pacientes entrevistados responderam a pergunta sobre qual o seu estado origem 59\% deste são do estado de São Paulo, Maranhão 7\%, Bahia 7\%, Santa Catarina 6\%, Minas Gerais 5\%, Mato Grosso do Sul 5\%, Espirito Santo 5\%, Amazonas $4 \%$, Goiás $1 \%$, Paraná $1 \%$, Piaúi $1 \%$.

Os diagnósticos das doenças prevalentes nos entrevistados foram: Leucemia Linfóide Aguda 26\%, Leucemia Mielóide Aguda 24\%, Mieloma Múltiplo 18\%, Síndrome Mielodisplásica 12\%, Anemia aplásica Severa 6\%, Linfoma Não Hodgkin 4\%, Doença de Hodgkin 2\% e Leucemia Linfóide Crônica $1 \%$. 
Tabela 1. Respostas referentes a cada item da escala de Herth. Jaú, São Paulo, 2021.

\begin{tabular}{|c|c|c|c|c|c|c|c|c|c|c|c|c|}
\hline \multirow[t]{2}{*}{ Alternativa } & \multicolumn{2}{|c|}{ Herth - Q1 } & \multicolumn{2}{|c|}{ Herth - Q2 } & \multicolumn{2}{|c|}{ Herth - Q3 } & \multicolumn{2}{|c|}{ Herth - Q4 } & \multicolumn{2}{|c|}{ Herth - Q5 } & \multicolumn{2}{|c|}{ Herth - Q6 } \\
\hline & PRÉ & PÓS & PRÉ & PÓS & PRÉ & PÓS & PRÉ & PÓS & PRÉ & PÓS & PRÉ & PÓS \\
\hline Discordo Compl. & $0 \%$ & $2 \%$ & $0 \%$ & $3 \%$ & $50 \%$ & $56 \%$ & $3 \%$ & $0 \%$ & $0 \%$ & $0 \%$ & $28 \%$ & $31 \%$ \\
\hline Discordo & $0 \%$ & $7 \%$ & $8 \%$ & $22 \%$ & $25 \%$ & $28 \%$ & $6 \%$ & $14 \%$ & $0 \%$ & $3 \%$ & $19 \%$ & $56 \%$ \\
\hline Concordo & $31 \%$ & $27 \%$ & $33 \%$ & $25 \%$ & $17 \%$ & $25 \%$ & $31 \%$ & $47 \%$ & $22 \%$ & $42 \%$ & $33 \%$ & $25 \%$ \\
\hline Concordo Compl. & $75 \%$ & $62 \%$ & $58 \%$ & $67 \%$ & $6 \%$ & $6 \%$ & $64 \%$ & $53 \%$ & $83 \%$ & $78 \%$ & $22 \%$ & $11 \%$ \\
\hline
\end{tabular}

\begin{tabular}{|c|c|c|c|c|c|c|c|c|c|c|c|}
\hline \multirow[t]{2}{*}{ Alternativa } & Herth $-Q 7$ & \multicolumn{2}{|c|}{ Herth $-Q 8$} & \multicolumn{2}{|c|}{ Herth $-Q^{9}$} & \multicolumn{2}{|c|}{ Herth $-Q 10$} & \multicolumn{2}{|c|}{ Herth - Q11 } & \multicolumn{2}{|c|}{ Herth $-Q 12$} \\
\hline & PRÉ PÓS & PRÉ & PÓS & PRÉ & PÓS & PRÉ & PÓS & PRÉ & PÓS & PRÉ & PÓS \\
\hline Discordo Compl. & $3 \%$ & $0 \%$ & $3 \%$ & $0 \%$ & $0 \%$ & $0 \%$ & $0 \%$ & $0 \%$ & $0 \%$ & $0 \%$ & $3 \%$ \\
\hline Discordo & $0 \%$ & $17 \%$ & $19 \%$ & $3 \%$ & $0 \%$ & $6 \%$ & $17 \%$ & $3 \%$ & $3 \%$ & $3 \%$ & $8 \%$ \\
\hline Concordo & $36 \% \quad 44 \%$ & $22 \%$ & $36 \%$ & $25 \%$ & $33 \%$ & $36 \%$ & $39 \%$ & $17 \%$ & $31 \%$ & $22 \%$ & $31 \%$ \\
\hline Concordo Compl. & $61 \%$ & $61 \%$ & $58 \%$ & $78 \%$ & $81 \%$ & $64 \%$ & $61 \%$ & $83 \%$ & $83 \%$ & $81 \%$ & $81 \%$ \\
\hline
\end{tabular}

Fonte: Autores (2021).

Nota-se que as afirmações 5 ("Eu tenho uma fé que me conforta"), 9 ("Eu me sinto capaz de dar e receber afeto/amor"), 11 ("Eu acredito no valor de cada dia") e 12 ("Eu sinto que minha vida tem valor e utilidade") obtiveram os maiores percentuais de resposta "Concordo Completamente", tanto no pré como no pós. Em relação às afirmações 3 e 6 , que possuem score invertido, chama-se atenção a distribuição das respostas à afirmação 6 ("Eu tenho medo do meu futuro"), grande parte dos pacientes responderam "Discordo completamente", mas as respostas foram bem distribuídas, com 55\% (pré) e $36 \%$ (pós) das respostas nas categorias que indicam concordância.

O Gráfico 1, demonstra o resultado em relação a questão 6 que obteve uma média de 2,7, inferior a esperada com valores próximo a 4. As menores médias foram das afirmações 3 ("Eu me sinto muito sozinho (a)") com média de 3,2 e afirmação 8 ("Eu me sinto muito forte"), média de 3,3 indicando que alguns dos pacientes se sentem sozinhos e/ou fracos. 
Gráfico 1. Média das informações referentes a cada item escala de Herth. Jaú, São Paulo, 2021.

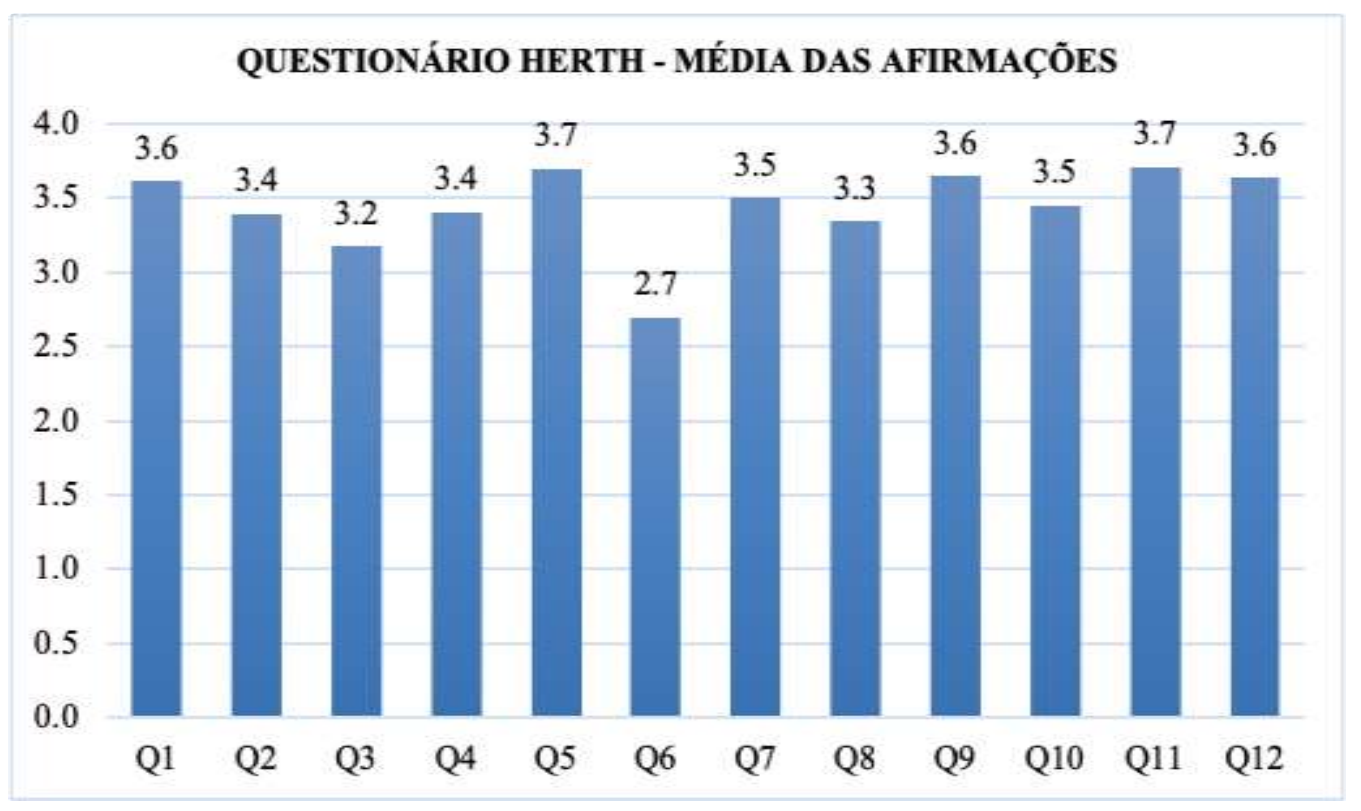

Fonte: Autores (2021).

Apenas 4 pacientes obtiveram score menor que 30, indicando um nível de esperança inferior aos demais, como demonstra o Gráfico 2.

Gráfico 2. Score total apresentados pelos pacientes. Jaú, São Paulo, 2021.

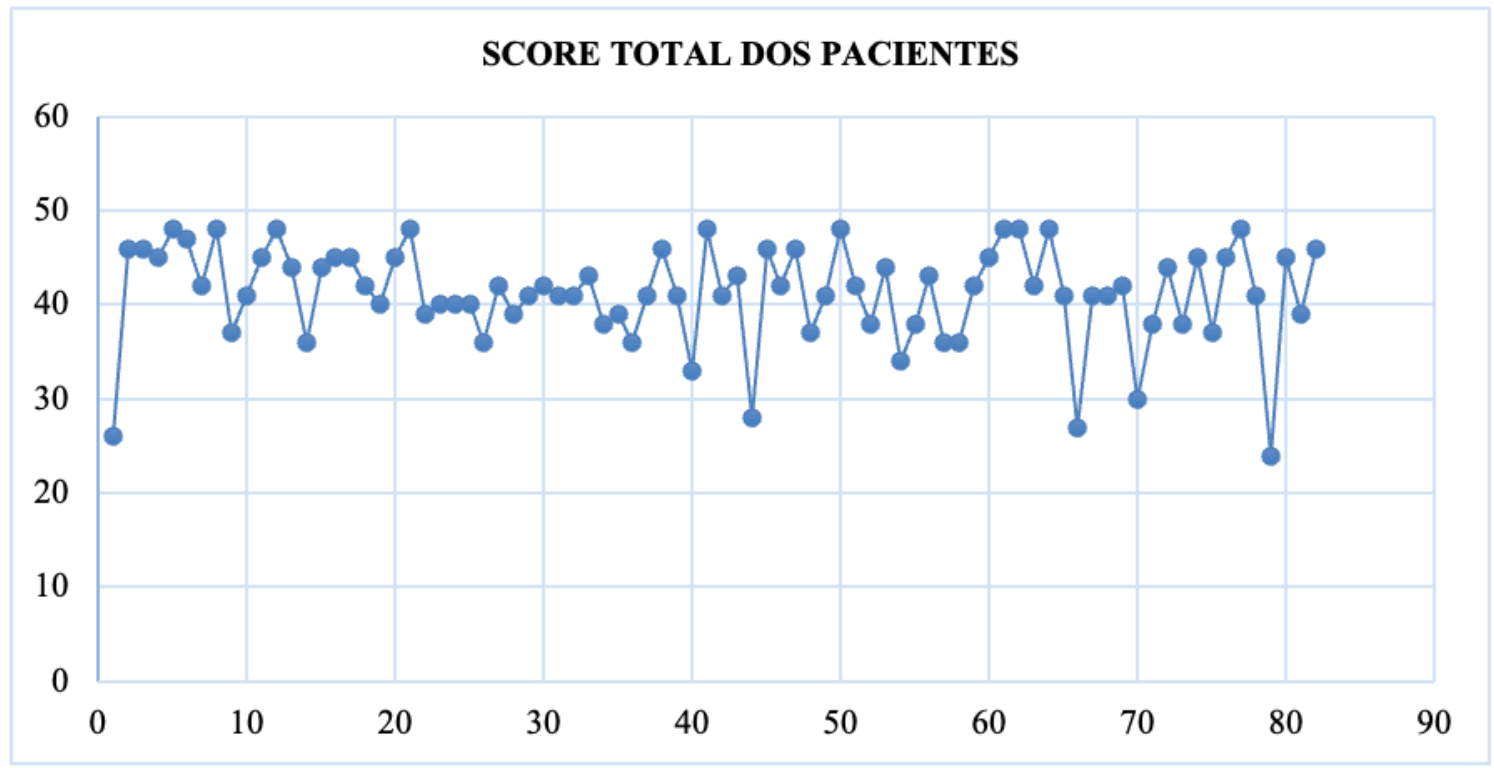

Fonte: Autores (2021).

Percebe-se que a grande maioria dos pacientes $(65,8 \%)$ obtiveram scores acima de 40, indicando alto nível de esperança. 
Gráfico 3. Abaixo apresenta-se, a distribuição desses scores:

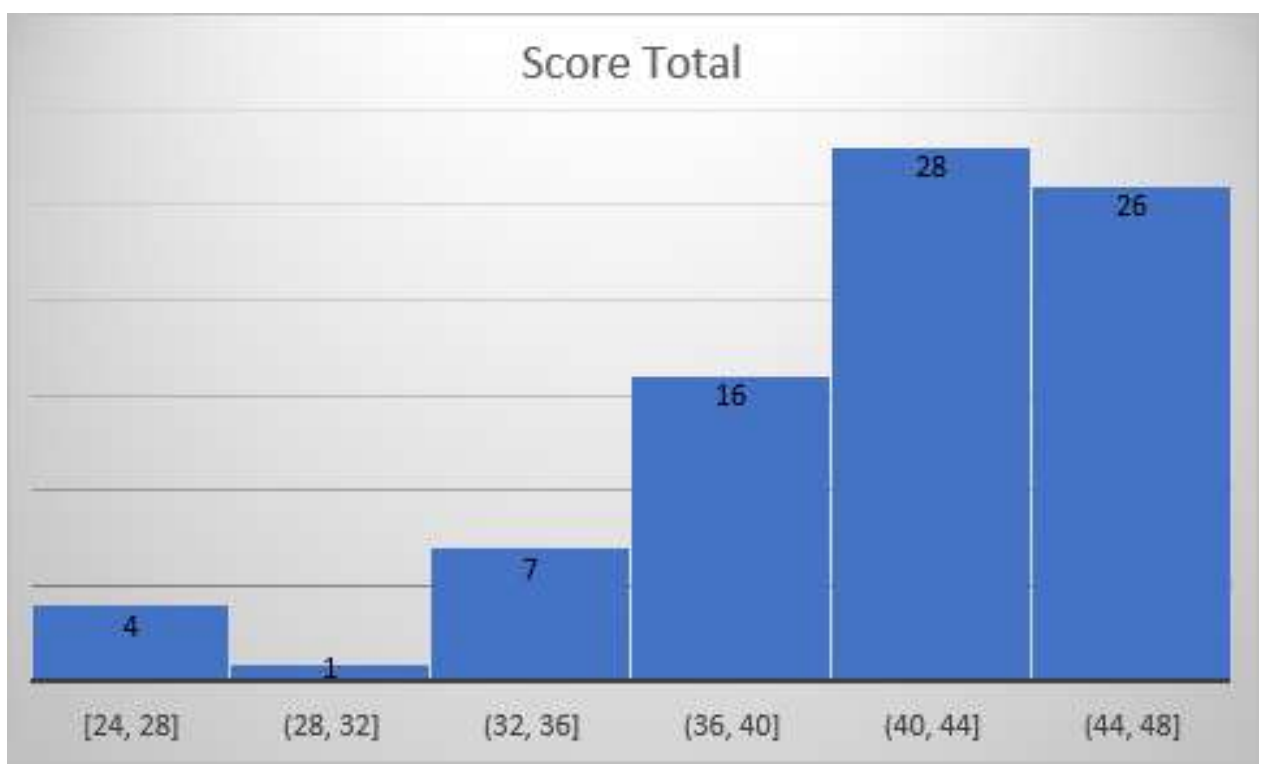

Fonte: Autores (2021).

Após a aplicação do teste de Spearman para verificar a existência de correlação entre as variáveis quantitativas (score total, tempo de seguimento - em meses, tempo de diagnóstico - em meses, idade, número de filhos e $\mathrm{N}^{\circ}$ de pessoas que residem na mesma casa).

Na matriz de p-valores acima, os destacados em amarelo foram os casos em a correlação foi considerada significativa (p-valor $\leq$ 0,05). Portanto, pode-se dizer que existe correlação entre o tempo de seguimento e tempo de diagnóstico (em meses), a idade e o tempo de diagnóstico e também entre idade e $\mathrm{n}^{\circ}$ de filhos.

A variável Score Total não apresentou correlação significativa com nenhuma dessas variáveis.

A Matriz de Correlação foi utilizada para verificar a intensidade e o sentido da correlação (positiva ou negativa). Os valores podem ser interpretados da seguinte maneira, na Tabela 2 :

Tabela 2. Correlação e verificação de intensidade e o sentido da correlação (positiva ou negativa). Jaú, São Paulo, 2021.

\begin{tabular}{ll}
\hline & Se $0,9 \leq|r|<1,0 \rightarrow$ há uma correlação muito forte \\
& Se $0,7 \leq|r|<0,9 \rightarrow$ há uma correlação forte \\
\hline & Se $0,4 \leq|r|<0,7 \rightarrow$ há uma correlação moderada; \\
\hline & Se $0,2 \leq|r|<0,4 \rightarrow$ há uma correlação fraca; \\
& Se $0<|r|<0,2 \rightarrow$ a correlação é muito fraca \\
\hline
\end{tabular}

Fonte: Autores (2021).

Sobre a correlação ser positiva ou negativa, pode-se dizer que um resultado positivo indica que conforme uma variável cresce a outra também cresce, e um resultado negativo indica que conforme uma variável cresce a outra diminui. 
Tabela 3. Respostas referentes a cada item da escala de Herth. Jaú, São Paulo, 2021.

\begin{tabular}{c|cccccc}
\hline & \multicolumn{4}{c}{ Matriz de Correlação } \\
& SCORE_TOTAL & Tempo Seguimento & Tempo Diagnóstico & Idade & Filhos & $\boldsymbol{N}^{\boldsymbol{o}}$ de pessoas na casa \\
\hline SCORE_TOTAL & 1,00 & $-0,10$ & $-0,10$ & $-0,17$ & $-0,07$ & 0,15 \\
Tempo Seguimento & $-0,10$ & 1,00 & 0,70 & $-0,01$ & 0,09 & $-0,06$ \\
Tempo Diagnóstico & $-0,10$ & 0,70 & 1,00 & $-0,23$ & $-0,10$ & $-0,01$ \\
Idade & $-0,17$ & $-0,01$ & $-0,23$ & 1,00 & 0,65 & $-0,10$ \\
Filhos & $-0,07$ & 0,09 & $-0,10$ & 0,65 & 1,00 & 0,16 \\
No de pessoas na casa & 0,15 & $-0,06$ & $-0,01$ & $-0,10$ & 0,16 \\
\hline
\end{tabular}

Fonte: Autores (2021).

Como exemplo, a correlação existente entre o tempo de seguimento e o tempo de diagnóstico $(0,70)$ é uma correlação forte e positiva, ou seja, os pacientes que demoraram mais tempo para ter o transplante foram os que também tiveram os tratamentos mais demorados. O mesmo para Idade e $n^{\circ}$ de filhos, conforme aumenta a idade, mais filhos possui, só que neste caso a correlação é moderada.

Já no caso de idade e tempo de diagnóstico a correlação é fraca e negativa, então, no caso desta amostra, idades maiores implicam em tempo de diagnóstico menores.

Gráfico 4. A relação entre os tempos pode ser visualizada no diagrama de dispersão abaixo:

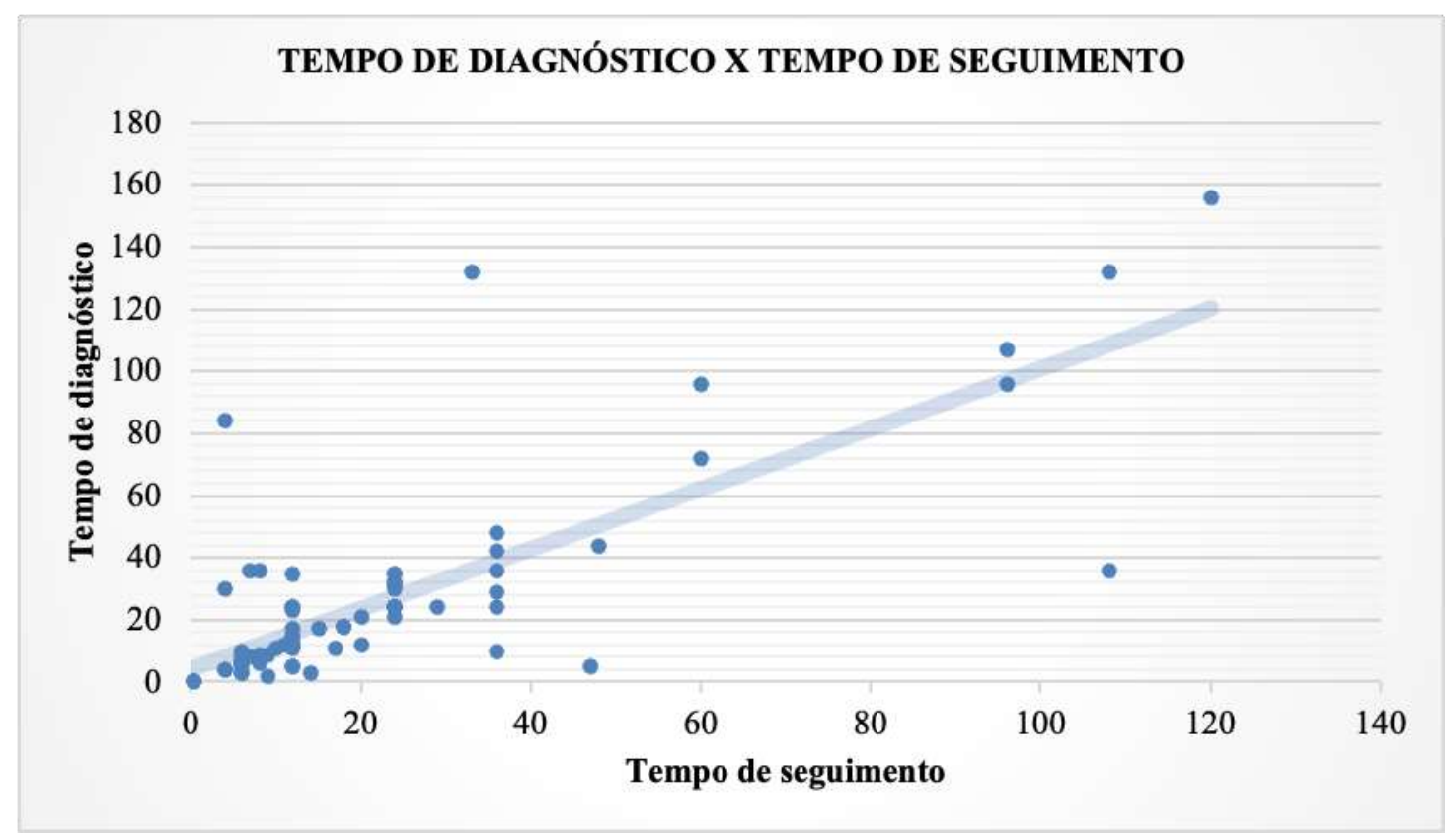

Fonte: Autores (2021).

Com análise e aplicação do teste de associação - Teste Qui-Quadrado, para testar a existência de associação entre o escore total da escala Herth e as variáveis categóricas, transformamos as variáveis categóricas em numéricas. Os valores foram então distribuídos nas seguintes categorias: 
Foi encontrada associação significativa entre o Score e a variável "Prática religiosa" (p-valor = 0,0288).

Gráfico 5. A relação entre prática religiosa e score total, havendo cruzamento dessas duas variáveis no gráfico abaixo:

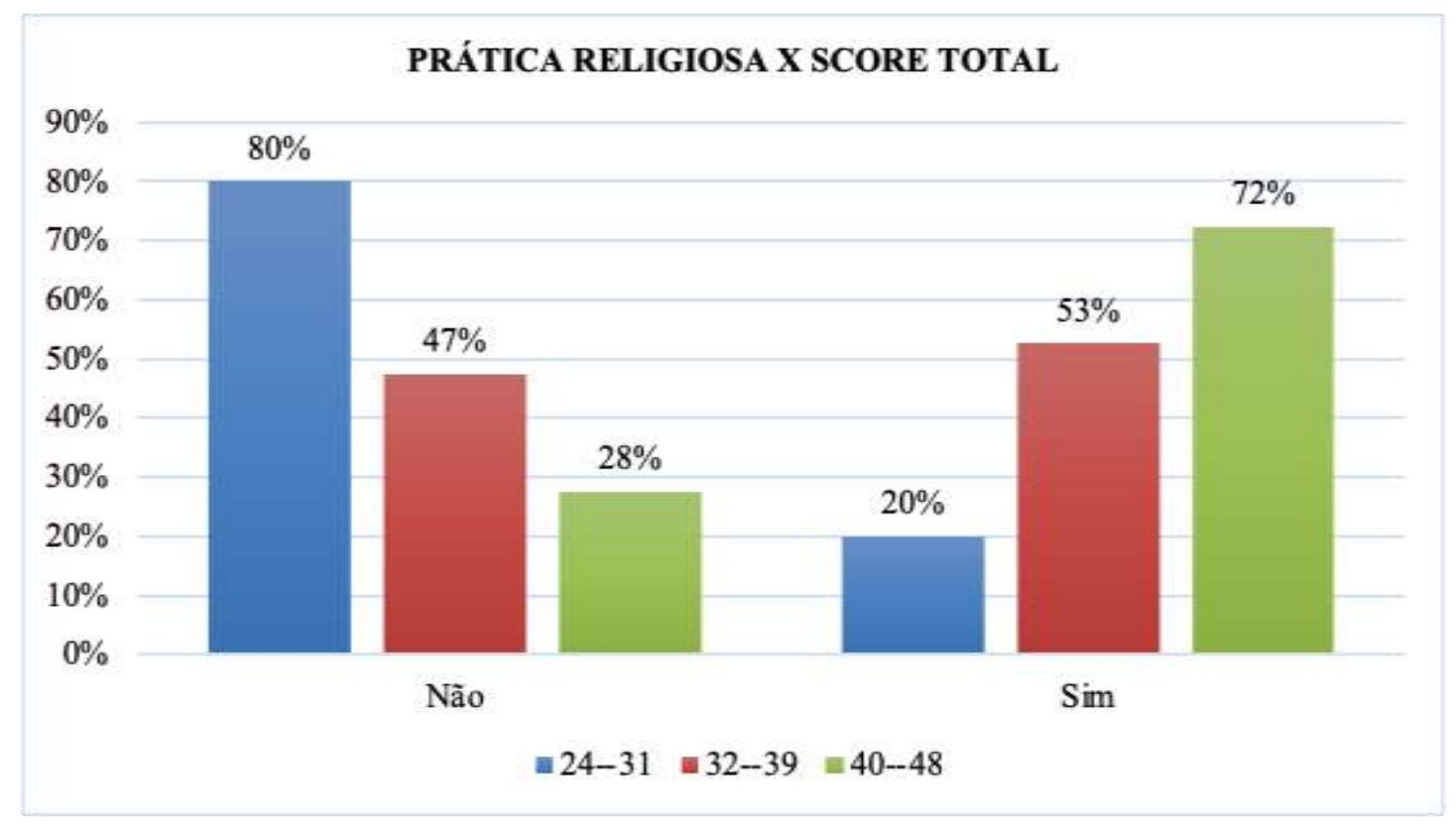

Fonte: Autores (2021).

Nota-se que, de modo geral, os religiosos foram os que apresentaram scores maiores (entre 40 e 48 - barra cinza), portanto, maiores níveis de esperança. E os não religiosos apresentaram scores menores (barra azul).

Após todas as etapas já realizadas, comparamos os scores dos dois grupos de respondentes (pré e pós-transplante). Como a variável é quantitativa discreta não é possível aplicar um teste de normalidade, sendo assim é necessário usar o teste Wilcoxon que é um teste não paramétrico para comparar os dois grupos. Observou-se que não existe diferença significativa, pois o p-valor foi de 0,5438 .

Observou-se que o nível de esperança era semelhante nos grupos, apresentando o grupo pré somente 1,2 pontos acima da média do grupo pós. Sendo assim, pode-se concluir que os dois grupos tiveram perspectivas semelhantes quando ao nível de esperança.

A escala de esperança de Herth é composta por 12 itens descritos de forma afirmativa e a graduação dos mesmos ocorre por escala tipo Likert de 4 pontos, variando de concordo completamente, indicado por 4, a discordo completamente, apontado por 1 . Os itens três e seis possuem o escore invertido. O escore total varia de 12 a 48 , sendo que quanto maior o escore, mais alto o nível de esperança.

Estudos têm evidenciado que esperança tem se tornado cada vez mais uma crença fundamental para impulsionar o indivíduo a agir, mover-se e alcançar algo que se busca. Desse modo a falta de esperança, torna-o mais denso, sem metas ou objetivos, aguardando a morte, assim deixando-os vulneráveis ao surgimento de depressão e iatrogenias (Ferreira \& Garcia, 2021).

No entanto a esperança é considerada como principal responsável por dar significado e alegria e sentido à vida, estudos validam e afirmam que a esperança, se origina da fé em deus ou de um quociente de espiritualidade que cada indivíduo traz em si (Silva et al., 2021). Desse modo, este estudo, buscou avaliar e descrever os diferentes graus de esperança em pacientes hematológicos, durante o diagnóstico e tratamento, pré e pós TCTH. 
Contudo, foi possível identificar diferentes níveis de esperança dos pacientes, podendo conferir subsídios, para o planejamento de cuidados mais adequado, com vista às ações mais apropriadas no estímulo à esperança e na redução do impacto da doença no cotidiano de cada paciente com câncer. Neste estudo os níveis de esperança encontrados mostraram os scores acima de 40 pontos. Esses resultados são próximo a de estudos realizados com uma média de 40 e 46, onde pacientes em tratamento antineoplásico apresentaram escore de 40,5 pontos na mesma escala (Carvalho et al., 2021).

Em relação aos itens da EEH foi possível identificar que as afirmações "Eu tenho uma fé que me conforta", "Eu me sinto capaz de dar e receber afeto/amor", "Eu acredito no valor de cada dia" e "Eu sinto que minha vida tem valor e utilidade" obtiveram os maiores percentuais de resposta "Concordo Completamente", em ambos os grupos de pacientes.

Os pacientes que possuem a fé como principal componente espiritual, tornando-o valioso para manutenção do bemestar, auxiliando numa perspectiva satisfatória para o enfrentamento e proporcionando satisfação de vida. A demais, isso indica a possiblidade de se relacionar com Deus e com forças transcendentais por meio de orações para reforçar a perspectiva de melhora de saúde e qualidade de vida no futuro, o que denota, também, tolerar 27 características estressoras vividas no momento presente, ao longo do tratamento ou até mesmo pós o tratamento (Belo et al., 2020).

Um dos aspectos encontrado em outras literaturas frente à esperança de vida é a questão do fator relacionado à prática religiosa, em nosso estudo os religiosos foram os que apresentaram scores maiores (entre 40 e 48), portanto, maiores níveis de esperança. E os não religiosos apresentaram scores menores (entre 24 e 31). Segundo Costa et al., (2019), a forma como cada indivíduo emana sua espiritualidade está diretamente relacionada às suas estratégias de enfrentamento, sendo a fé em Deus um sentimento expresso em situações complexas e desfavoráveis. Nesse caso, a religiosidade e a espiritualidade, através da fé, permitem o ajustamento do paciente diante do câncer, podendo ele extrair pensamentos positivos das experiências estressantes, a fim de ocasionar uma mudança de vida. Aliados às crenças religiosas e espirituais, altos níveis de esperança proporcionam satisfação e bem-estar, elevando a resiliência diante de doenças que ameaçam a vida.

Os estudos apontam que não tem uma justificativa específica para a questão da religião, mas sim ao aspecto que está relacionado à espiritualidade cada crença vem com seus conceitos, princípios e doutrinas diferentes, sendo que o que predomina é a fé, não importa o tipo de religião em que o indivíduo segue, mas sim se lhe faz bem, ele vai à procura de apoio emocional e esperança de alcançar os seus objetivos. Foi observado que algumas pessoas acreditam que alguém está olhando por elas, uma força que vem do sobrenatural, o que caracteriza a crença individual e particular (Dib et al., 2020; Silva et al., 2019).

Um dos scores com menor grau de pontuação na EEH está correlacionado as afirmações "Eu tenho medo do meu futuro", que obteve uma média de 2,7, inferior a esperada com valores próximo a 4, as afirmações "Eu me sinto muito sozinho (a)" e "Eu me sinto muito forte", também apresentaram médias inferiores ao esperado indicando que alguns dos pacientes se sentem sozinhos e/ou fracos. Além disso, este estudo traz que a maioria dos pacientes entrevistados e que estão enfrentando a doença, são de 11 estados diferentes, sendo a grande maioria cerca de (59\%) pertencente ao estado de São Paulo, e (41\%) de outros estados do Brasil. Segundo Mourão et al., (2017), o convívio familiar é um fator de proteção importante durante esse processo saúde e doença, tendo em conta que o convívio social está interligado aos menores índices de carga de estresse e sentimento de solidão. No entanto na unidade de TCTH, essas vivências são intensificadas devido às complicações inerentes ao processo em que o paciente será submetido, onde estará sujeito a inúmeras restrições, como é o caso da internação em condição de isolamento, onde o 28 isolamento protetor acaba sendo considerado um fator que impacta significativamente no cotidiano do paciente, permanecendo restrito do seu meio social com o objetivo de evitar possíveis infecções e demais complicações (Santos et al., 2017). Somado a isso, outras situações estressoras encontradas neste estudo foram os efeitos adversos do tratamento, tempo de internação em decorrências de diversas complicações, intercorrências infecciosas, doença do enxerto contra o hospedeiro (DECH) além da necessidade de suporte de cuidados intensivos. Em um estudo realizado por 
Santos et al., (2017), as complicações são baseadas em virtude de todo o processo do regime de condicionamento com altas doses de quimioterapia, os efeitos adversos do TCTH estão presentes durante o tratamento.

Desse modo, é relevante refletir sobre a atuação dos profissionais inseridos no processo de cuidar de pacientes sob tratamento para transplante de células tronco-hematopoiéticas, buscando programar ações de caráter subjetivo, como a escuta ativa, para colher queixas e compreender os sentimentos que possam comprometer os mais diversos níveis de esperança (Costa et al., 2019).

Em relação ao fator idade e tempo de diagnóstico o nosso estudo mostrou que a correlação é fraca e negativa, então, no caso desta amostra, idades maiores implicam em tempo de diagnóstico menores. Desse modo o diagnóstico precoce se torna imprescindível além de aumentar de forma significativa a sobrevida global de pacientes com diagnóstico de câncer e outras doenças graves, do mesmo modo que aumenta as possibilidades de tratamento. Ainda assim, muitos pacientes ainda são diagnosticados em atendimentos de emergência, o que aumenta a probabilidade de que essas doenças já estarem em estádios mais avançados, o que contribui para redução da sobrevida e pior experiência no tratamento da doença (Dominguez et al., 2020; Moreira \& Silva Borges, 2020).

\section{Conclusão}

Conclui-se que os pacientes em tratamento pré e pós transplante de células tronco-hematopoiéticas apresentaram altos níveis de esperança durante todo o processo de tratamento. Entretanto, foi mostrado que os indivíduos que realizam prática religiosa como estratégia para o enfrentamento da doença, apresentaram altos níveis de esperança.

Este estudo mostra-se relevante e evidência a importância dos profissionais de enfermagem, pois por estarem em contato direto com o paciente e seus familiares ao longo de todo o tratamento, eles possuem a oportunidade de estabelecer uma relação em que se torna possível detectar as necessidades religiosas e espirituais do aspecto paciente e familiar cuidador. Desse modo, o cuidado voltado ao aspecto da espiritualidade será direcionado ao indivíduo, respeitando-se sua concepção de mundo, o que trará benefícios ao utilizar positivamente suas estratégias de enfrentamento, manter e elevar a sua esperança.

Por tanto, sugere-se que sejam realizados mais estudos que busquem demonstrar a relação entre os níveis de esperança e o coping religioso, de pacientes em pré e pós transplante de células tronco-hematopoiéticas, em diferentes momentos ao longo do tratamento, de modo a identificar os possíveis fatores que influenciem esses níveis de esperança e consequentemente, promover saúde numa perspectiva holística.

\section{Referências}

Belo, F. M. P., Albuquerque, M. C. dos S. de., Santos, R. C. S., Melo-Neto , V. L. de., Lima, J. L. R. de., Maynart, W. H. da C., Ornelas, A. C. C. de., \& Alves, V. de M. (2020). Desesperança e transtornos mentais em profissionais de enfermagem de serviços oncológicos. Research, Society and Development, 9(11), e60491110065. https://doi.org/10.33448/rsd-v9i11.10065.

Carvalho, P. P., Souza, D. C. de, Rossato, L., \& Scorsolini-Comin, F. (2021). Acolhimento da religiosidade/espiritualidade em intervenções psicológicas no hospital: Relato de experiência. Research, Society and Development, 10(13), e520101321606. https://doi.org/10.33448/rsd-v10i13.21606.

Costa, D. T., Silva, D. M. R. D., Cavalcanti, I. D. L., Gomes, E. T., Vasconcelos, J. L. D. A., \& Carvalho, M. V. G. D. (2019). Coping religioso/espiritual e nível de esperança em pacientes com câncer em quimioterapia. Revista Brasileira de Enfermagem, 72, 640-645. https://doi.org/10.1590/0034-7167-2018-0358.

Dib, R. V., Gomes, A. M. T., Ramos, R. de S., França, L. C. M., \& Marques, S. C. (2020). O câncer e suas representações sociais para pacientes oncológicos. Research, Society and Development, 9(9), e187997134. https://doi.org/10.33448/rsd-v9i9.7134.

Dominguez, R. G. S., Freire, A. S. V., Silva, S. S., \& Campos, N. A. S. (2020). Morbimortalidade por doenças oncohematológicas no Brasil entre 2002 e 2016. Revista Eletrônica Acervo Saúde, 12(10), e3795-e3795. https://doi.org/10.25248/reas.e3795.2020.

Eloia, S. M. C., Ximenes, M. A. M., Eloia, S. C., Galindo Neto, N. M., Barros, L. M., \& Caetano, J. Á. (2021). Enfrentamento do coping religioso e esperança na doença renal crônica: estudo controlado randomizado. Revista da Escola de Enfermagem da USP, 55. https://doi.org/10.1590/1980-220X-REEUSP-20200368 .

Ferreira, J. C. M., \& Garcia, R. R. (2021). Narrativas de vida de pacientes oncológicos sob cuidados paliativos: o olhar das peças poéticas. Research, Society and Development, 10(11), e583101119919. https://doi.org/10.33448/rsd-v10i11.19919. 
Franco, A. de A., Anjos, B. F. dos., Ribeiro, W. A., Oliveira, A. T. de., Monsores, A. F., Dias, L. L. da C., Ranauro, K. C. D. S. S., \& Macedo, G. F. (2021). Sistematização da assistência de enfermagem no cuidado com a mulher mastectomizada: Uma revisão integrativa. Research, Society and Development, 10(9), e31710918121. https://doi.org/10.33448/rsd-v10i9.18121.

Freitas, T. F. D., de Souza, S. R., do Espírito Santo, F. H., Tocantins, F. R., \& do Couto, L. L. (2021). A família e suas demandas para o autocuidado apoiado no contexto da doença onco-hematológica. Research, Society and Development, 10(2), e29110212527-e29110212527. https://doi.org/10.33448/rsdv10i2.12527.

Gomes, I. M., Lacerda, M. R., Hermann, A. P., Rodrigues, J. A. P., Zatoni, D. C. P., \& Tonin, L. (2019). Cuidados realizados pelo familiar cuidador da criança em pós-transplante de células-tronco hematopoiéticas. Revista Latino-Americana de Enfermagem, 27. https://doi.org/10.1590/1518-8345.2298-3120.

Grandizoli, M. V., dos Santos Júnior, R., Ibiapina, I. S. M., \& Garcia, V. C. B. (2017). Indicadores de esperança, ansiedade e depressão de pacientes em tratamento oncológico. Arquivos de Ciências da Saúde, 24(3), 65-70. https://doi.org/10.17696/2318-3691.24.3.2017.718.

Li, P., Guo, Y. J., Tang, Q., \& Yang, L. (2018). Eficácia da intervenção de enfermagem para aumento da esperança em pacientes com câncer: uma metaanálise. Revista Latino-Americana de Enfermagem, 26. https://doi.org/10.1590/1518-8345.1920.2937.

Lima, C. G. D., Lacerda, G. M. D., de Beltrão, I. C. S. L., de Araújo Alves, D., \& Albuquerque, G. A. (2020). Impacto do Diagnóstico e do Tratamento do Câncer de Mama em Mulheres Mastectomizadas. Ensaios e Ciência C Biológicas Agrárias e da Saúde, 24(4), 426-430. https://doi.org/10.17921/14156938.2020v24n4p426-430.

Menezes, R. R., Kameo, S. Y., da Cunha Santos, D. K., de Almeida, K. A., Santos, L. P., de Jesus Santos, L., \& Mocó, G. A. A. (2021). Esperança de vida de pessoas com câncer acompanhadas pela Atenção Primária à Saúde. Research, Society and Development, 10(4), e12510413644-e12510413644. https://doi.org/10.33448/rsd-v10i4.13644.

Moreira, R. A., \& Silva Borges, M. D. (2020). Perfil e nível de esperança entre pacientes que realizam hemodiálise e diálise peritoneal. Cogitare Enfermagem, 25. http://dx.doi.org/10.5380/ce.v25i0.67355.

Mourão, C. M. L., Fernandes, A. F. C., Moreira, D. P., \& Martins, M. C. (2017). Entrevista motivacional no suporte social de cuidadores de pacientes com câncer de mama em quimioterapia. Revista da Escola de Enfermagem da USP, 51. https://doi.org/10.1590/S1980-220X2017001803268.

Okuma, G. Y., Manhães, M. F. M., Pedras, R. N., De Domenico, E. B. L., \& Bergerot, C. D. (2021). Espiritualidade, religiosidade, distress e qualidade de vida em pacientes oncológicos. Revista Psicologia e Saúde, 13(2), 3-17. https://doi.org/10.20435/pssa.v13i2.1097.

Polit, D. F. \& Beck, C. T. (2019). Fundamentos de pesquisa em enfermagem: Avaliação de Evidências Para Práticas de Enfermagem. 9. ed. Porto Alegre: Artmed, cap. 9, p.135-159.

Santos, D. D. F. K., de Camargo, M. J. G., dos Santos, D. R., \& Lolatto, G. A. (2017). O uso de estratégias de coping de pacientes adultos submetidos ao transplante de células tronco hematopoéticas. Revista Ocupación Humana, 17(2), 20-33. https://doi.org/10.25214/25907816.169.

Sartore, A. C., \& Grossi, S. A. A. (2008). Escala de Esperança de Herth: instrumento adaptado e validado para a língua portuguesa. Revista da Escola de Enfermagem da USP, 42(2), 227-232. http://doi.org/10.1590/S0080-62342008000200003.

Schuster, J. T., Feldens, V. P., Iser, B. P. M., \& Ghislandi, G. M. (2015). Esperança e depressão em pacientes oncológicos em um hospital do sul do Brasil. Rev AMRIGS, 59(2), 84-9.

Sette, C. P., \& Capitão, C. G. (2018). Investigação do suporte social e qualidade de vida em pacientes com câncer. Saúde e Pesquisa, 11(1), 151-162. https://doi.org/10.17765/1983-1870.2018v11n1p151-162.

Silva, N. M., Santos, M. A. D., Oliveira, R. A. A. D., Storti, L. B., Souza, I. M. O., Formighieri, P. F., \& Marques, S. (2019). Idosos em Tratamento Quimioterápico: Relação entre Nível de Estresse, Sintomas Depressivos e Esperança. Psicologia: Teoria e Pesquisa, 35 . https://doi.org/10.1590/0102.3772e35441.

Silva, D. D., Neto, J. Z., Dóro, M. P., \& Menegatti, C. L. (2021). Revisão narrativa sobre a avaliação psicológica em pacientes pré-transplante de célulastronco hematopoiéticas. Brazilian Journal of Development, 7(2), 16048-16069. https://doi.org/10.34117/bjdv7n2-298.

Tempesta, O. (2015). O significado da esperança. https://www.a12.com/redacaoa12/espiritualidade/o-significado-da-esperanca. Não paginado.

Wakiuchi, J., Marchi, J. A., Norvila, L. S., Marcon, S. S., \& Sales, C. A. (2015). Esperança de vida de pacientes com câncer submetidos à quimioterapia. Acta Paulista de Enfermagem, 28, 202-208. https://doi.org/10.1590/1982-0194201500035. 